OPEN ACCESS

Edited by:

Patrícia Maciel,

University of Minho, Portugal

Reviewed by:

Sokol V. Todi,

Wayne State University, United States

Carla S. Lopes,

University of Porto, Portugal

*Correspondence:

Taro Ishiguro

Ishiguro.nuro@tmd.ac.jp

Yoshitaka Nagai

yoshi.nagai@med.kindai.ac.jp

Kinya Ishikawa

piconuro@tmd.ac.jp

†Present address:

Yoshitaka Nagai,

Department of Neurology, Faculty of Medicine, Kindai University,

Osakasayama, Japan

Specialty section:

This article was submitted to

Neurodegeneration,

a section of the journal

Frontiers in Neuroscience

Received: 31 December 2020

Accepted: 31 March 2021

Published: 25 May 2021

Citation:

Ishiguro T, Nagai $Y$ and Ishikawa $K$ (2021) Insight Into Spinocerebellar

Ataxia Type 31 (SCA31) From

Drosophila Model.

Front. Neurosci. 15:648133.

doi: 10.3389/fnins.2021.648133

\section{Insight Into Spinocerebellar Ataxia Type 31 (SCA31) From Drosophila Model}

\author{
Taro Ishiguro ${ }^{1 *}$, Yoshitaka Nagai ${ }^{2 * t}$ and Kinya Ishikawa ${ }^{1,3 *}$ \\ ${ }^{1}$ Department of Neurology and Neurological Science, Tokyo Medical and Dental University, Bunkyo City, Japan, \\ ${ }^{2}$ Department of Neurotherapeutics, Osaka University Graduate School of Medicine, Suita, Japan, ${ }^{3}$ Department \\ of Personalized Genomic Medicine for Health, Graduate School, Tokyo Medical and Dental University, Bunkyo City, Japan
}

Spinocerebellar ataxia type 31 (SCA31) is a progressive neurodegenerative disease characterized by degeneration of Purkinje cells in the cerebellum. Its genetic cause is a 2.5- to 3.8-kb-long complex pentanucleotide repeat insertion containing (TGGAA)n, (TAGAA)n, (TAAAA)n, and (TAAAATAGAA)n located in an intron shared by two different genes: brain expressed associated with NEDD4-1 (BEAN1) and thymidine kinase 2 (TK2). Among these repeat sequences, (TGGAA)n repeat was the only sequence segregating with SCA31, which strongly suggests its pathogenicity. In SCA31 patient brains, the mutant BEAN1 transcript containing expanded UGGAA repeats (UGGAA exp) was found to form abnormal RNA structures called RNA foci in cerebellar Purkinje cell nuclei. In addition, the deposition of pentapeptide repeat (PPR)

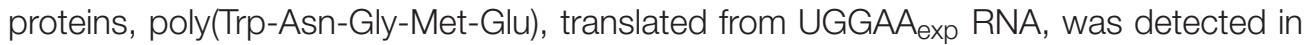
the cytoplasm of Purkinje cells. To uncover the pathogenesis of UGGAA exp in SCA31, we generated Drosophila models of SCA31 expressing UGGAA exp RNA. The toxicity

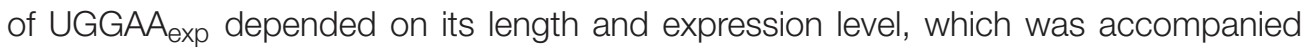
by the accumulation of RNA foci and translation of repeat-associated PPR proteins in Drosophila, consistent with the observation in SCA31 patient brains. We also revealed that TDP-43, FUS, and hnRNPA2B1, motor neuron disease-linked RNAbinding proteins bound to UGGAA exp RNA, act as RNA chaperones to regulate the formation of RNA foci and repeat-associated translation. Further research on the role of RNA-binding proteins as RNA chaperones may also provide a novel therapeutic strategy for other microsatellite repeat expansion diseases besides SCA31.

Keywords: RBP, TDP-43, RNA chaperone, RNA foci, RAN translation, microsatellite repeat, SCA31

\section{INTRODUCTION}

More than 40 refractory neurological diseases are caused by microsatellite repeat expansions, including spinal and bulbar muscular atrophy; Huntington disease (HD); Friedreich ataxia; fragile $\mathrm{X}$ syndrome; fragile X-associated tremor/ataxia syndrome (FXTAS); myotonic dystrophy types 1 and 2 (DM1, DM2); C9orf72-associated amyotrophic lateral sclerosis/frontotemporal dementia (C9orf72-ALS/FTD); dentatorubral-pallidoluysian atrophy; spinocerebellar ataxia (SCA) types 1, 2, 3, 6, 7, 8, 10, 12, 17, 31, 36, and 37 (Paulson, 2018); and benign adult familial myoclonic epilepsy 
(Ishiura et al., 2018). Microsatellite repeats are tandem stretches of three to six nucleotides in the genome that are often polymorphic in length. At some genetic loci, microsatellite repeats become genetically unstable (Pearson et al., 2005; Nelson et al., 2013; Banez-Coronel and Ranum, 2019) and cause disease when the length of the repeat sequence exceeds a certain threshold. This size threshold differs for each repeatharboring gene. In affected individuals, large repeat expansions show somatic and intergenerational instabilities and lead to various disease phenotypes. Disease-causing repeat expansion mutations can reside in both coding and noncoding regions of the respective gene, including the promoter region, $5^{\prime}$ UTR, an alternate exon, $3^{\prime}$ UTR, and an intron. The lengths of repeat expansion in noncoding regions are usually much greater and more unstable than those in coding regions. The molecular pathogenesis of these repeat expansion diseases is considered to include loss of host gene function as in fragile $\mathrm{X}$ syndrome and Friedreich ataxia, production of toxic RNAs as in DM1 and C9orf72-ALS/FTD, and production of toxic polypeptides as in polyglutamine diseases such as $\mathrm{HD}$ and several SCAs. The complexity of their pathogenesis has gradually been recognized: the loss of host gene function and the production of both toxic RNA and polypeptides all seem to play roles in a single disease, as exemplified by the pathogenesis of HD. The repeat expansions in some of these diseases are transcribed into aberrant repeat RNAs, which can form hairpin and G-quadruplex structures and RNA foci that interact with RNA-binding proteins (RBPs) and alter their activities, which would lead to dysfunction in RNA metabolism (Mohan et al., 2014; Liu et al., 2017; Zhang and Ashizawa, 2017; Reddy et al., 2020). For example, the depletion of key splicing regulatory RBPs such as muscleblind-like 1 (MBNL1) and CUG-binding protein 1 (CUGNP1) sequestered in RNA foci can induce pre-mRNA missplicing in DM1. Furthermore, expanded repeat RNAs undergo repeat-associated non-ATG (RAN) translation to generate toxic repeat proteins in all reading frames ( $\mathrm{Zu}$ et al., 2011, 2013). Taking these findings together, the microsatellite repeat expansions trigger a wide range of pathogenic pathways, which leads to a variety of neurological and neurodegenerative diseases.

\section{OVERVIEW OF SCA31: CLINICAL PERSPECTIVE AND FEATURES}

SCA31 is one of the autosomal-dominant neurodegenerative disorders showing a relatively pure cerebellar form of ataxia. It is typically a disease of late adulthood, with onset peaking between 60 and 65 years of age (Nagaoka et al., 2000; Sato et al., 2009; Nakamura et al., 2017; Ishikawa and Nagai, 2019). SCA31 is a common ataxia in Japan (Onodera, 2006; Ouyang et al., 2006; Basri et al., 2007; Hayashi et al., 2007; Nozaki et al., 2007; Yoshida et al., 2009), whereas it is very rare in surrounding parts of Asia such as Korea, Taiwan, and China (Lee et al., 2007, 2012; Ouyang et al., 2012; Pedroso et al., 2015) and extremely rare in Caucasian populations (Ishikawa et al., 2011). SCA31 patients were previously found in Brazil, but all of them were descendants of Japanese immigrants (Pedroso et al., 2015), which suggests that SCA31 shows a strong founder effect.

SCA31 is caused by a 2.5 - to $3.8-\mathrm{kb}$ insertion of complex pentanucleotide repeats containing (TGGAA)n, which is located in overlapping introns of the BEAN1 and TK2 genes on chromosome 16q22.1 (Sato et al., 2009). The insertion sequence is a complex pentanucleotide repeat containing (TGGAA)n, (TAGAA)n, (TAAAA)n, and (TAAAATAGAA)n (Figure 1). Importantly, the length of this insertion was shown to be inversely correlated with the age at onset in SCA31 patients. Among healthy controls, the vast majority (99.7\%) were found to have a short TAAAA repeat of only 8-20 repeats at this locus, although very rare large insertions of (TAAAA)n, (TAGAA)n, and (TAAAATAGAA)n stretch (approximately 1.5$2.0 \mathrm{~kb}$ in size) lacking (TGGAA) sequences were found in Japanese controls $(0.23 \%)$. Therefore, (TGGAA)n was considered to be the only causative repetitive sequence among these repeats segregating with the phenotype in SCA31. In addition to these repeats, the SCA31 mutation site is associated with diverse pentanucleotide repeats such as (TACAA)n, (GAAAA)n, (TAACA)n, (TGAAA)n, and (TAAAA)n. While (TAAAA)n is the prototypical pentanucleotide repeat in humans, (TGGAA)n and (TAGAA)n repeats are exclusively found in the Japanese, whereas (TACAA)n, (GAAAA)n, (TAACA)n, and (TGAAA)n pure repeat expansions are found in Caucasians (Ishikawa et al., 2011). Among these repeats, (TGGAA)n is the only repetitive sequence that segregates with cerebellar neurodegeneration.

\section{NEUROPATHOLOGICAL FEATURES OF SCA31}

In patients with SCA31, marked degeneration of the cerebellar cortex with Purkinje cell-predominant neuronal loss was observed in the cerebellum (Owada et al., 2005). Purkinje cells often showed shrinkage of the cell body. In addition, they were often surrounded by halo-like amorphous materials, which were shown to be positively stained with anti-calbindinD-28 K (CaBP28K) and anti-synaptophysin antibodies in immunohistochemistry (Ishikawa and Mizusawa, 2010; Yoshida et al., 2014). Therefore, these materials were considered to consist mainly of the somatic sprouts of Purkinje cells and presynaptic terminals innervated from basket cells, neurons of the inferior olivary nucleus, or other neurons that connect with Purkinje cells.

A neuropathological hallmark of microsatellite repeat expansion diseases is the intracellular accumulation of mutant RNA containing an expanded nucleotide repeat as RNA foci in the nucleus and/or cytoplasm of affected cells. This has emerged as an important disease mechanism for the growing class of such diseases (Wojciechowska and Krzyzosiak, 2011; Zhang and Ashizawa, 2017). Previous studies identified RNA foci within the nuclei of SCA31 Purkinje cells labeled with an oligonucleotide probe against UGGAA repeats (Sato et al., 2009; Niimi et al., 2013).

Several lines of evidence indicate that RAN translated proteins are involved in a growing number of microsatellite repeat expansion diseases, including HD, DM1, SCA8, FXTAS, and 


\section{SCA31 insertion sequence}

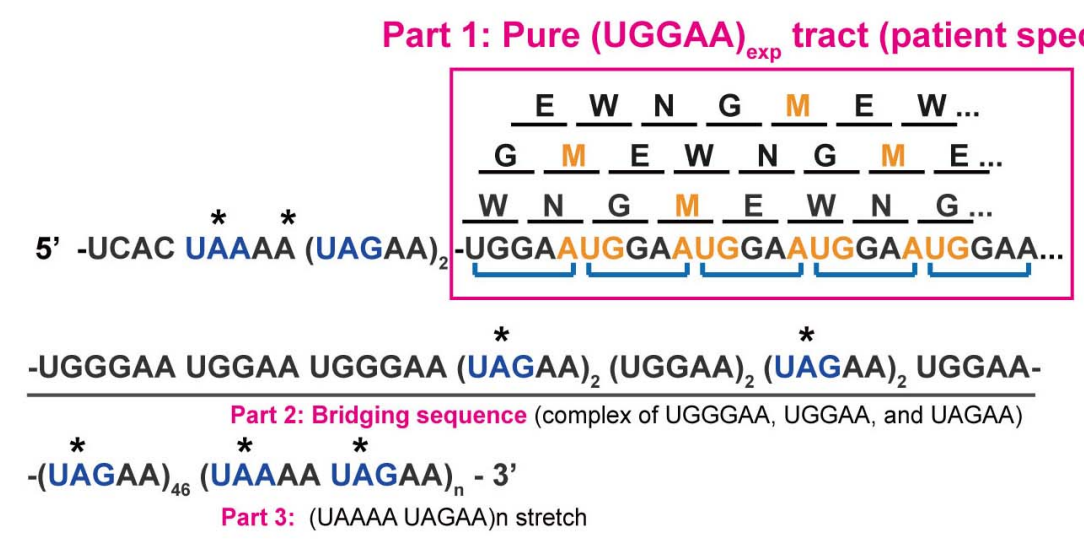

The sequence of the insertion in control

-UCAC (UAAAA) $)_{2}$ AAA (UAAAA) $)_{3-5}$ AA (UAAAA) ${ }_{2}$ TA TAAAA TAGAA TAAAA -(UAGAA) (UAAAA UAGAA)

The (UGGAA)n was not detected
UGGAA repeat
Haripin structure

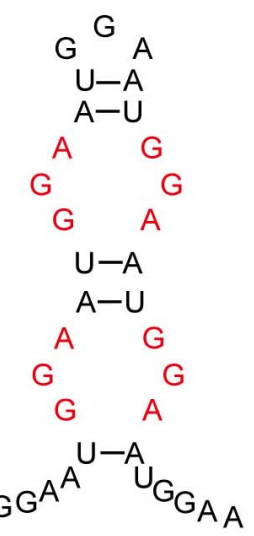
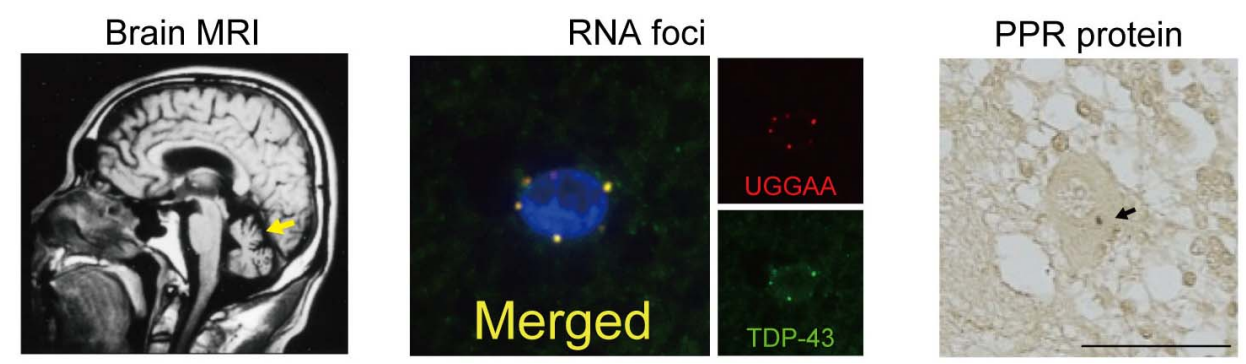

FIGURE 1 | Schematic diagram of PPR proteins. Amino acids are shown as single letters. Translation of the UGGAA repeat in all frames would result in the production of an identical PPR protein, poly-WNGME. Asterisk (*) indicates a stop codon in the sequence surrounding the UGGAA repeat. Note that the TGGAA repetitive sequence itself includes an AUG translation-initiation codon, and both the TAAAA and the TAGAA sequences flanking the TGGAA repetitive sequence include UAA and UAG translation stop codons. AUG codons shown in orange stand for start, and blue color codons stand for stop in repeat sequence in a BEAN1 transcript. SCA31 shows slowly progressive cerebellar ataxia and MRI-proven cerebellar atrophy. Purkinje cells are primarily affected showing characteristic degenerative changes. Abnormal RNA aggregates called RNA foci are occasionally detected in Purkinje cell nuclei by fluorescent in situ hybridization (FISH) using probes against the (UGGAA)n-containing transcripts (Ishiguro et al., 2017). Furthermore, TDP-43 clearly colocalized with RNA foci in human SCA31 Purkinje cells. PPR proteins were also detected in cell bodies and dendrites of SCA31 Purkinje cells (arrows) (Ishiguro et al., 2017). Possible hairpin structures in UGGAA repeat containing internal loop with three consecutive G-A, G-G, and A-G mismatch base pairs (shown in red) (Shibata et al., 2021).

C9orf72-ALS/FTD (Banez-Coronel and Ranum, 2019; Nguyen et al., 2019). Unlike other microsatellite repeat expansion diseases, it is important to note that, in SCA31, the UGGAA repetitive sequence itself includes an AUG translation-initiation codon (Ishiguro et al., 2017). On the other hand, several repetitive sequences in the SCA31 locus found in normal populations, including UAAAA, UAGAA, UGAAA, and UAACA, contain UAA, UAG, and UGA translation stop codons, and thus these repeats are unlikely to be translated. Translation of the UGGAA repeat in any frame would result in a single pentapeptide repeat (PPR) protein, poly-WNGME. Another special circumstance in SCA31 is that the UGGAA repeat is flanked by putative stop codons UAA and UAG (Figure 1), which suggests that, if the PPR is expressed, the translation has to occur within this sequence. In fact, anti-PPR antibodies detected granular structures in cell bodies and dendrites of SCA31 Purkinje cells, but not in control brains, which suggests that PPR proteins are expressed in SCA31 brains where (UGGAA) exp is expressed by BEAN1 transcription. Taking these findings together, TGGAA pentanucleotide repeat expansion gives rise to toxic UGGAA repeat RNAs, which form RNA foci and are translated into poly(WNGME) proteins in Purkinje cells in SCA31.

Much of the previous progress in our understanding of various microsatellite repeat expansion diseases suggests the multifaceted nature of the underlying pathogenic mechanisms in SCA31. The first pathogenic mechanism of SCA31 suggested by nuclear and cytoplasmic RNA foci containing UGGAA $\mathrm{UEx}_{\text {exp }}$ transcripts is RNAmediated toxicity, as these foci might sequester RNA-binding proteins and consequently alter RNA metabolism, as in other repeat expansion disorders (Sato et al., 2009; Niimi et al., 2013; 
Ishiguro et al., 2017). Another distinctive feature of SCA31 is the deposition of PPR proteins, mostly in the cytoplasm of patients' Purkinje cells: poly(WNGME) is synthesized from the UGGAA repeat transcripts. Repeat-associated AUG or non-AUG (RAN) translation is proposed to be the mechanism responsible for PPR protein production (Ishiguro et al., 2017). Note that the TGGAA repetitive sequence itself includes an AUG translationinitiation codon. Haploinsufficiency of the causative gene is also a potential disease-causing mechanism in microsatellite repeat expansion diseases; however, the expression of BEAN1, TK2, and nearby genes in SCA31 patient brains is not reduced by the insertion of complex repeats in this locus, which indicates that haploinsufficiency is unlikely to be a primary mechanism behind the disease. The expanded repeat can be transcribed in both sense and antisense directions, resulting in sense and antisense RNA foci or protein aggregates in several repeat expansion diseases including SCA8, HDL2, and C9ALS/FTD (Moseley et al., 2006; Wilburn et al., 2011; Gendron et al., 2013; Zu et al., 2013). Considering that the insertion is located in introns of BEAN1 and TK2 transcribed in opposite directions in SCA31, TK2 transcripts including extended repeats may behave in a similar manner. The function of this transcript and its toxicity remains to be explored. To elucidate the molecular mechanisms of SCA31, we employed Drosophila to model SCA31 expressing UGGAA repeat in BEAN1 direction as it has been productively used to study neurodegenerative diseases in recent years (McGurk et al., 2015; Şentürk and Bellen, 2018). Insights from Drosophila models of SCA31 are discussed below.

\section{Drosophila AS A GENETIC MODEL FOR SCA31}

We have established Drosophila models of SCA31 expressing expanded UGGAA repeats to analyze the gain of toxic function, in a manner mostly similar to that used for other repeat expansion diseases (Ishiguro et al., 2017). Eighty to 100 copies of TGGAA repeats together with three other repeat components, (TAGAA)n, (TAAAA)n, and (TAAAATAGAA)n, derived from SCA31 patients were subcloned into the pUAST vector, which has an upstream activating sequence (UAS) element to allow spatial control of target gene expression in tissue-specific GAL4 driver lines, and transgenic flies carrying TGGAA repeats were generated. Control fly lines were also established expressing control repeat tract lacking (TGGAA)n found in the normal population. In both transiently and stably expressed cultured cell models, the (UGGAA)n in the BEAN1 transcripts yields more toxicity than control transcripts and forms RNA foci (Niimi et al., 2013). The contribution of the UGGAA repeat tract to disease pathogenesis in vivo was first evaluated in the Drosophila models

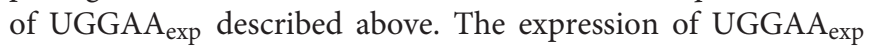
(80-100 repeats) RNA in compound eyes using the GMRGAL4 driver caused severe eye degeneration, depending on its expression level, whereas control repeat and short UGGAA 22 RNA showed no detectable degeneration.

The expression of $\mathrm{UGGAA}_{\exp }$ in the nervous system with elav-Gal4 driver exhibited a shorter lifespan and progressive locomotor defects, whereas control repeat and $\mathrm{UGGAA}_{22}$ did not. As expected, RNA fluorescence in situ hybridization (FISH) revealed remarkable accumulation of UGGAA exp $_{\text {RNA }}$ as RNA foci in eye imaginal disks of UGGAA $\mathrm{Uxp}_{\text {-expressing }}$ flies, consistent with the pathology of SCA31 patients. Fly lines expressing control repeat or $\mathrm{UGGAA}_{22}$ had no detectable RNA foci in the fly tissue. In this model, PPR protein was also detected by Western blot and immunohistochemistry, and its expression level was positively correlated to the severity of eye degeneration in Drosophila. Therefore, both UGGAA exp $_{\text {p }}$ RNA toxicity and PPR protein toxicity may contribute to the neurodegeneration in SCA31.

\section{TDP-43 DISENTANGLES UGGAA exp $_{\text {RNA }}$ FROM RNA FOCI FORMATION}

RNA-binding proteins are essential at all levels of RNA processing, including transcription, alternative splicing, stabilization, degradation, and RNA granule formation, in both the nucleus and the cytoplasm. The loss of RBPs influences the processing of these noncoding RNAs and contributes to global RNA dysregulation. Considering that proteins sequestered in RNA foci have been reported to play an important role in gain of toxic function of microsatellite repeat expansion diseases (Zhang and Ashizawa, 2017), the interacting partners of repeat RNA have been studied extensively in SCA31 using pull-down approaches (Ishiguro et al., 2017).

Interestingly, several ALS/FTD-linked RBPs were identified as UGGAA-binding proteins, such as TDP-43 (TAR DNAbinding protein, $43 \mathrm{kDa}$ ), FUS, and hnRNPA2B1. Indeed, FISH analysis showed the colocalization of TDP-43 with RNA foci in the nucleus of human SCA31 Purkinje cells. Surprisingly,

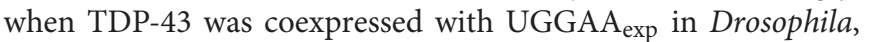

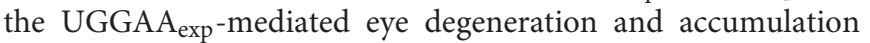
of RNA foci were dramatically suppressed. Conversely, the knockdown of TDP-43 enhanced eye degeneration and the formation of RNA foci. Subsequent analysis of atomic force microscopy and circular dichroism spectroscopy demonstrated that the binding of TDP-43 to UGGAA repeat RNA alters its structure. Thus, TDP-43 functions as an RNA chaperone for UGGAA repeat RNA, consistent with the definition of an RNA chaperone (Figure 2; Rajkowitsch et al., 2007). Notably, as mentioned earlier, PPR proteins are also expressed in Drosophila tissue and SCA31 brain. TDP-43 coexpression with expanded UGGAA in the fly was shown to dramatically inhibit the expression and accumulation of PPR protein in fly tissue. In addition to TDP-43, coexpression of FUS or hnRNPA2B1 markedly suppressed eye degeneration in UGGAA exp-expressing flies. These RBPs mitigated the deposition of both RNA foci and PPR proteins without causing degradation of the UGGAA exp $_{\text {. }}$ RNA transcripts, confirming their function as RNA chaperones. TDP-43 and hnRNPA2B1 also have a protective role against CGG-repeat mediated toxicity in Drosophila model of FXTAS (He et al., 2014). Interestingly, it is suggested that TDP-43 suppression of CGG repeat toxicity is dependent on interaction with hnRNPA2B1 orthologs in an FXTAS Drosophila model. 


\section{Myotonic dystrophy}
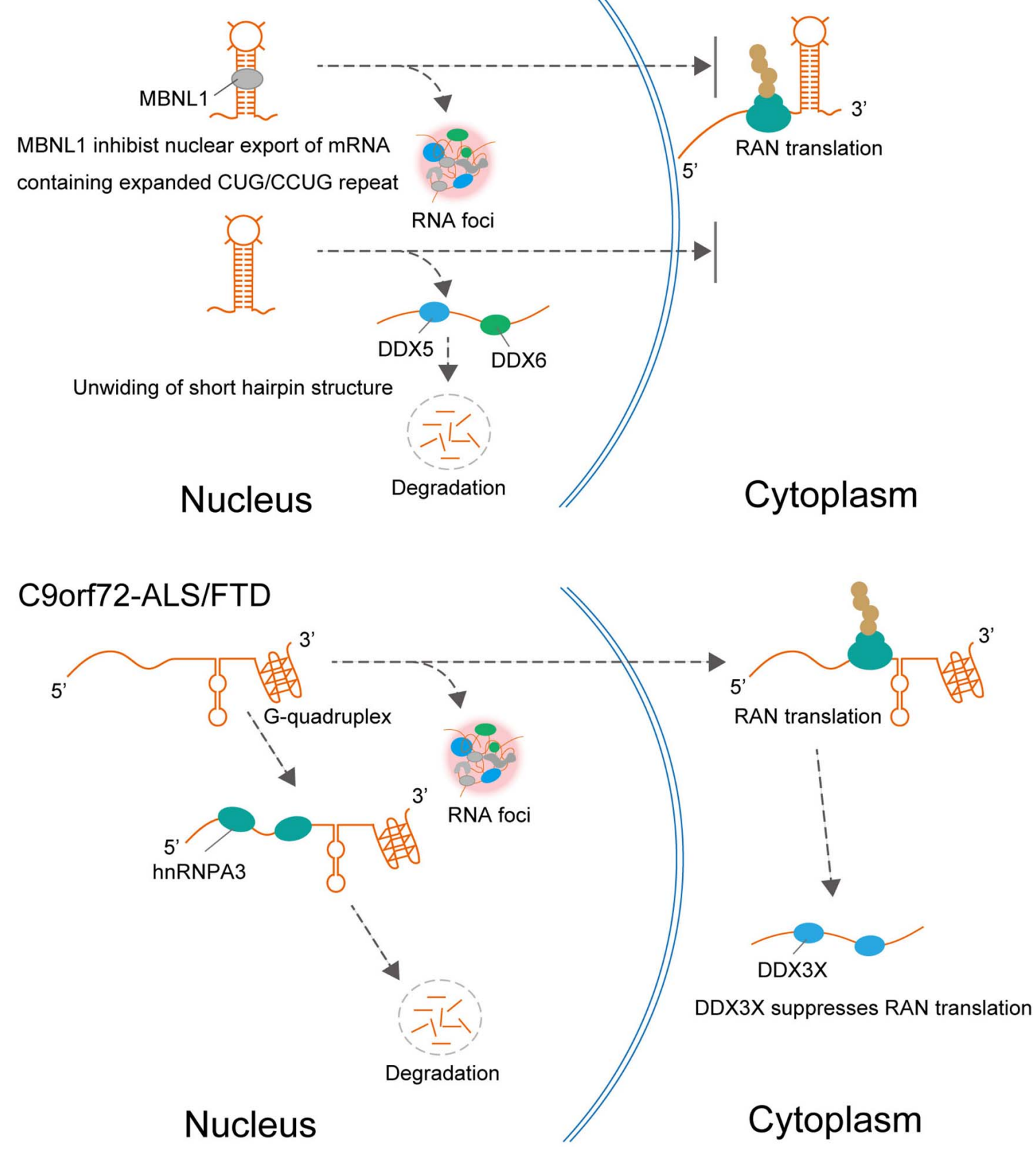

FIGURE 2 | In DM1, MBNL1 promotes nuclear retention of expanded CUG repeat RNA and RNA foci formation, resulting in repression of RAN translation from CUG repeat RNA. In addition to MBNL1, DDX5, and DDX6 are also involved in DM1. DDX5 leads to degradation of the expanded CUG and CCUG RNAs and suppresses RNA foci formation. In C9orf72-ALS/FTD, hnRNPA3 reduced repeat RNA expression levels leading to decreasing RNA foci formation and DPR deposition. The helicase DDX3X, which unwinds (or relaxes) RNA, suppresses RAN translation and toxicity.

Overexpression of TDP-43 suppresses CGG repeat-mediated missplicing of hnRNPA2/B1-targeted transcripts. Note that TDP-43, FUS, and hnRNPA2B1 act independently to suppress UGGAA repeat-mediated toxicity in Drosophila model of SCA31. These results suggest that the rescuing effect of TDP-43 for FXTAS could be different from that for SCA31. Pur $\alpha$ is an RBP identified to interact with CGG repeat in Drosophila model of FXTAS (Jin et al., 2007). Overexpression of Puro suppresses CGG repeat-mediated neurodegeneration in Drosophila. Importantly, Pura is also found in the nuclear inclusions of FXTAS patient brains. These findings suggest that RNA-binding proteins that associate with repeat RNAs play an important role in the 
pathogenesis in repeat expansion diseases. Targeting factors critical for modulating RNA secondary structures could be potential therapeutic strategy in FXTAS and C9ALS/FTD as well. Knockdown of DDX3X, Dead-box helicase that is required for resolution of RNA-RNA structures and 43S PIC-mRNA binding and PIC scanning in GC-rich 5' UTR in FMR1, suppresses CGG repeat-mediated toxicity by inhibiting RAN translation (Linsalata et al., 2019). On the other hand, surprisingly, expression of DDX3X reduces endogenous DPR level and mitigates toxicity in C9ALS/FTD patient cells, and silencing of DDX3X enhances GGGGCC repeat-mediated toxicity in Drosophila of C9ALS/FTD (Figure 3; Cheng et al., 2019). Although DDX3X has opposing effects on RAN translation of GGGGCC repeat in C9ALS/FTD and CGG repeat in FXTAS, an RNA helicase that unwinds the repeat RNA structure would be expected to reduce RAN translation via modulation of RNA secondary structures. Taken together, targeting factors that modulate RNA secondary structures associated with RNA foci formation and RAN translation could be potential therapeutic strategies for SCA31 and related repeat expansion diseases.

Interestingly, these suppressive effects of TDP-43, FUS, and hnRNPA2B1 differ from those of other RBPs that interact with expanded repeat RNAs such as MBNL1 in DM1 and hnRNPA3 in C9orf72-ALS/FTD (Figure 2). For example, MBNL1 is sequestered in intranuclear inclusions containing expanded repeat RNA in the nucleus in DM1 and DM2, which leads to loss of MBNL function. In this process, MBNL1 promotes the nuclear retention of expanded CUG repeat RNA and the formation of RNA foci, resulting in the repression of RAN translation from CUG repeat RNA (Kino et al., 2015). It is also suggested that hnRNP $\mathrm{H}$ inhibits nuclear export of the expanded CUG RNA, which leads to its nuclear retention in DM1 (Kim et al., 2005). Therefore, several RBPs may play a protective role by

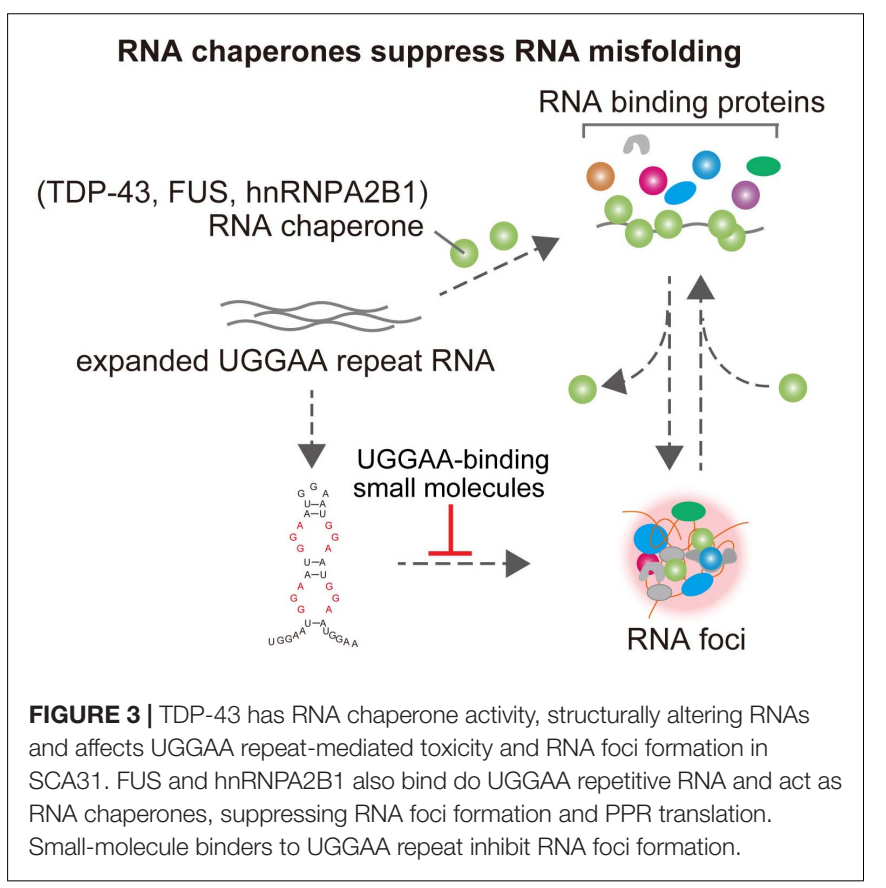

promoting mutant RNA nuclear retention and the formation of RNA foci, which could act as an RNA sink associated with RBPs. In addition to MBNL1 and hnRNPH, Staufen 1 and two members of the DEAD-box RNA helicase family, DDX5 and DDX6, are also involved in DM1. The protein expression level of DDX5 is reduced in DM1 biopsied muscle, and upregulation of DDX5 leads to degradation of the expanded CUG and CCUG RNAs and suppresses the formation of RNA foci (Pettersson et al., 2014; Jones et al., 2015). hnRNPA3 is one member of the heterogeneous nuclear ribonucleoprotein family, which specifically binds to the GGGGCC repeat RNA in the pathology of C9orf72-ALS/FTD (Mori et al., 2013). Unlike the protective role of RNA chaperones in SCA31, hnRNPA3 negatively regulates repeat RNA expression levels, which leads to decreased formation of RNA foci and DPR deposition (Mori et al., 2016).

\section{SMALL-MOLECULE BINDERS TO UGGAA REPEAT INHIBIT RNA FOCI FORMATION AND SUPPRESS EYE DEGENERATION IN Drosophila MODEL OF SCA31}

GGGGCC repeat RNA folds into a G-quadruplex and hairpin secondary structure in C9ALS/FTD, and CGG repeat RNA forms hairpin structure in FXTAS. Previous intensive research discovered that small molecules, which selectively bound GGGGCC or CGG repeat RNA, prevented sequestration of an $\mathrm{RBP}$ and inhibited RAN translation in vitro and in vivo models of C9ALS/FTD or FXTAS (Su et al., 2014; Simone et al., 2018; Green et al., 2019). These results suggest that targeting RNA secondary structures related to neurodegeneration could be a potential therapeutic strategy. UGGAA repeat is also suggested to fold into hairpin structure containing internal loop with three consecutive G-A, G-G, and A-G mismatch base pairs (Figure 1; Shibata et al., 2021). Recently, a chemical screen identified naphthyridine carbamate dimer (NCD) targeting disease-causing UGGAA repeat RNAs in SCA31 (Shibata et al., 2021). Structural analysis of the NCD-UGGAA/UGGAA complex by nuclear magnetic resonance spectroscopy revealed the mode of binding that recognizes four guanines in the UGGAA/UGGAA pentad by hydrogen bonding with four naphthyridine moieties of two NCD molecules. NCD disrupts RNA foci formation of UGGAA RNA by inhibiting interaction of UGGAA RNA and RBPs in vitro and ameliorates compound eye degeneration in the Drosophila model of SCA31 (Figure 3). These data provide proof of principle that targeting UGGGAA repeat harpin has therapeutic potential as well as G-quadruplex and hairpin secondary structure in C9ALS/FTD or FXTAS.

\section{BALANCING RNA-RBP CROSSTALK IS ESSENTIAL FOR RNP HOMEOSTASIS}

Although our SCA31 studies highlighted novel roles of TDP43, FUS, and hnRNPA2B1 as RNA chaperones for UGGAA $\mathrm{Uxp}_{\mathrm{exp}}$ 
RNA to modulate its folding and regulate the formation of toxic RNA aggregates (Figure 3), these RBPs on their own are known to play a toxic role in the pathogenesis of ALS/FTD (Ling et al., 2013). For example, pathological inclusions of TDP-43 can be found in the nucleus and cytosol of neurons and glia in sporadic and familial forms of ALS and FTD. Furthermore, patient-derived induced pluripotent stem cells and overexpression of TDP-43 in experimental animals recapitulated some of the pathological features of human ALS and FTD with neurodegeneration accompanied by various molecular phenotypes such as TDP-43 aggregation and aberrant RNA metabolism. Given that the UGGAA repeat RNA interacts with these RBPs, it is possible that such RBP toxicity may be ameliorated by binding RNAs. Against this background, flies expressing ALS-linked mutant TDP-43 G298S were crossed with $\mathrm{UGGAA}_{22}$ flies, which do not show any neurodegeneration. Although TDP-43 G298S-expressing flies exhibited severe compound eye degeneration, coexpression of $\mathrm{UGGAA}_{22}$ significantly suppressed the eye degeneration as well as mutant TDP-43 aggregation in eye imaginal disks. Furthermore, coexpression of $\mathrm{UGGAA}_{22}$ also suppressed the eye degeneration of flies expressing either FUS or mutant hnRNPA2B1 D290V, which suggests that UGGAA 22 buffers the propensity of these RBPs to aggregate (Ishiguro et al., 2017).
These results reveal that functional crosstalk of the RNARBP network regulates their own quality and homeostasis of ribonucleoprotein (RNP), which suggests that balancing the RNA-RBP crosstalk is a potential therapeutic approach for both microsatellite repeat expansion diseases and RBP proteinopathies.

Recent intensive studies have suggested that RBPs containing low complexity domains such as TDP-43, FUS, and hnRNPA2B1 can induce liquid-liquid phase separation in the process of formation of dynamic membrane-less organelles including Cajal bodies, paraspeckles, nuclear speckles, and stress granules (Weber and Brangwynne, 2012; Molliex et al., 2015; Courchaine et al., 2016). The combination of protein subdomains; RNA parameters such as concentration, structure, length, and sequence composition; and physiological salt conditions can greatly influence phase separation (Kroschwald et al., 2015; Jain and Vale, 2017; Maharana et al., 2018). Increasing the concentration of RBPs can diminish the liquid-like properties of these RNA granules, thereby promoting the formation of hydrogels and eventually an insoluble amyloidlike aggregate (Kato et al., 2012; Guo and Shorter, 2015; Shin and Brangwynne, 2017; Wolozin, 2019; Rhine et al., 2020). Conversely, the high RNA-to-protein concentration ratio in the nucleus buffers the phase separation of RBPs
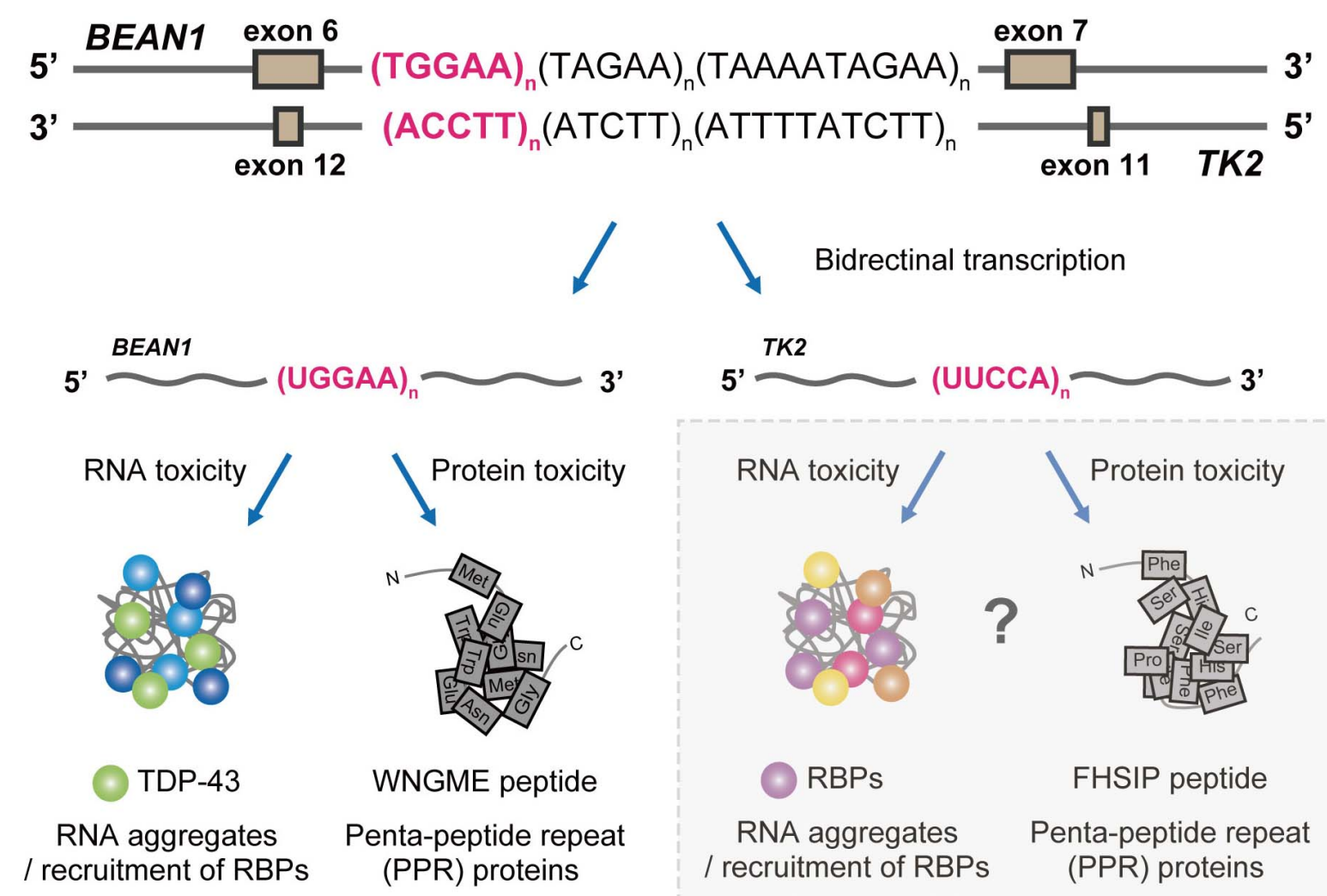

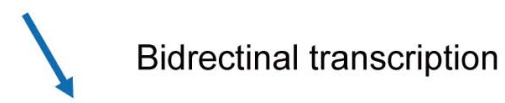
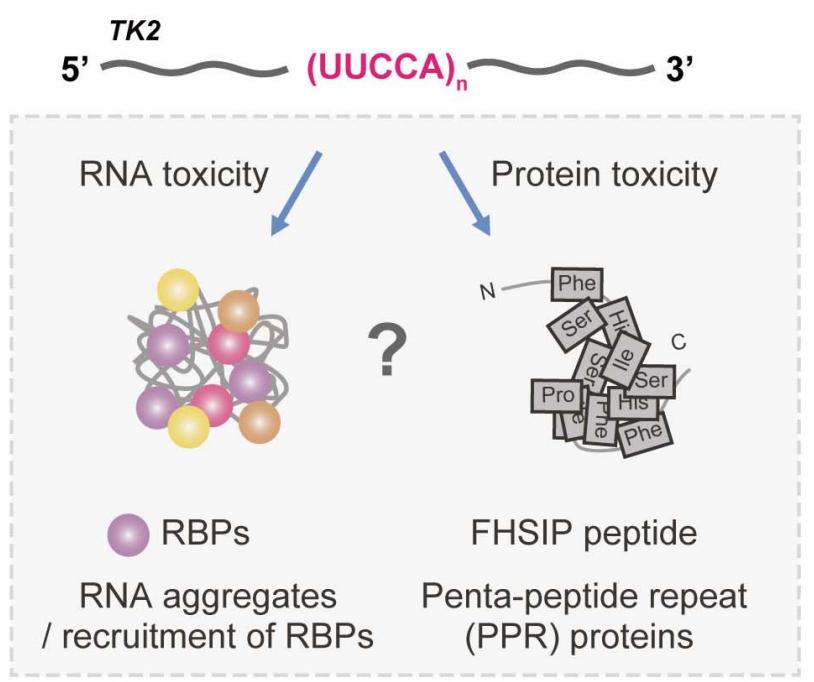

FIGURE 4 | Proposed pathogenic mechanisms in SCA31. BEAN1 transcripts including expanded UGGAA repeat and TK2 transcripts including expanded UUCCA repeat may cause neurodegeneration by RNA foci formation and RAN translation. Further investigation will clarify whether TK2 transcripts with the expanded repeat behave in a similar manner. 
such as FUS and TDP-43 (Gasset-Rosa et al., 2019). Taking these findings together, considering that balancing RNARBP crosstalk is essential for RNP homeostasis, both the RNA chaperone activity of RBPs and the buffering function of RNAs for target RBPs might be essential to regulate the formation of membrane-less organelles through proper liquid-liquid phase separation and the maintenance of these compartments.

\section{UGGAA $_{\text {exp }}$ IS A COMMON MODULAR STRUCTURE WITH SATELLITE III RNAS IN THE FORMATION OF nSBs}

From an in silico search of TGGAA pentanucleotide sequences in the human genome, TGGAA repeats are abundant in the centromeres of chromosomes 2, 4, 7, 10, 16, 17, 20, and Y. In addition, satellite III (SatIII) DNA in pericentromeric heterochromatin also has a modular structure composed of the TGGAA pentanucleotide repeats in common with SCA31 (Valgardsdottir et al., 2004). Notably, SatIII noncoding RNA containing UGGAA repeats is transcribed under heat stress to form nuclear stress bodies (nSBs) and plays an important role in regulating the splicing machinery by recruiting certain splicing factors such as serine/arginine-rich splicing factor 1 (SRSF1) and scaffold attachment factor B to nSBs (Metz et al., 2004; Valgardsdottir et al., 2008; Eymery et al., 2010). The recruitment of SRSF1 to nSBs occurs in a SatIII-dependent manner, and the downregulation of SatIII transcripts attenuates the heat shockinduced transcriptional repression of target genes. Conversely, the overexpression of SATIII transcripts results in the formation of $\mathrm{nSBs}$ and transcriptional repression, even without heat shock. Considering that SFRS1 and SFRS9 directly bind to UGGAA exp,

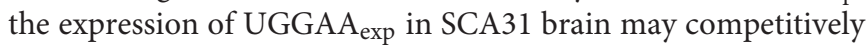
inhibit SatIII function, which leads to the misregulation of nSB formation and stress response. Indeed, the neuron-specific knockdown of Drosophila hsrw, functional analogs of SatIII, impairs locomotion in larval and adult flies, which implies that the dysfunction of SatIII mediated by UGGAA exp $_{\text {RNA in SCA31 }}$ could lead to neurodegeneration (Jolly and Lakhotia, 2006; Lo Piccolo and Yamaguchi, 2017; Lo Piccolo et al., 2019).

\section{CONCLUDING REMARKS}

In this article, we reviewed recent progress in our understanding of the pathogenesis of SCA31, mainly focusing on our recent findings revealed by Drosophila models. We demonstrated a novel role of repeat RNA-binding RBPs as RNA chaperones that modulate the repeat RNA misfolding and buffer its toxicity (Figure 3; Ishiguro et al., 2017).

Our study not only provided novel insights into the molecular pathogenesis of SCA31 and related repeat expansion diseases but also opened a new avenue toward the development of therapies for these diseases. Nevertheless, the presence of both RNA foci and PPR proteins in SCA31 still raises important questions about their relative contributions to the disease pathogenesis, and several questions still remain unanswered:

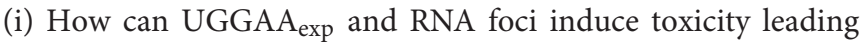
to neurodegeneration? (ii) Are PPR proteins necessary or sufficient to induce neurotoxicity? (iii) Do RBPs that bind to other disease-causing repetitive sequences such as C9orf72ALS/FTD, FXTAS, SCA36, or DM1 indeed play a protective role as RNA chaperones? (iv) Can TK2 transcripts including extended UUCCA repeats cause neurodegeneration with RNA foci formation and RAN translation? (Figure 4). It is important to determine whether UUCCA repeats could form RNA foci and sequester RBPs and produce poly(Phe-His-Ser-Ile-Pro, FHSIP) peptide in SCA31. We hope that genetic approaches taking advantage of Drosophila will help to address these important issues and contribute to establishing therapies for SCA31 and other repeat expansion diseases.

\section{AUTHOR CONTRIBUTIONS}

TI, YN, and KI designed the manuscript, assessed the literature, and edited the subsequent drafts and revisions. TI wrote the initial draft of the manuscript. All the authors contributed to the article and approved the submitted version.

\section{FUNDING}

This work was supported in part by a Grant-in-Aid for Scientific Research on Innovative Areas (Brain Protein Aging and Dementia Control) (17H05699 to YN) from the Ministry of Education, Culture, Sports, Science and Technology; by Grants-in-Aid for Scientific Research (A) (KI) and for Scientific Research (C) (B) (KI), and for Challenging Exploratory Research (24659438 to YN) from the Japan Society for the Promotion of Science; by Health and Labour Sciences Research Grants for Research on Development of New Drugs (H24Soyaku-Sogo-002 to YN) and on the Research Committee for Ataxic Diseases (H23-Nanchi-014 to YN) from the Ministry of Health, Labour and Welfare; by a grant for Practical Research Projects for Rare/Intractable Diseases (JP17ek0109102 and JP20ek0109302 to KI; JP16ek0109018, JP19ek0109222, and JP20ek0109316 to YN) and the Strategic Research Program for Brain Sciences (Integrated Research on Neuropsychiatric Disorders) (JP20dm0107061 to YN) from the Japan Agency for Medical Research and Development; and by Intramural Research Grants for Neurological and Psychiatric Disorders (27-7, 27-9, $30-3$, and 30-9 to YN) from the National Center of Neurology and Psychiatry, Mitsubishi Foundation, Natural Science (KI).

\section{ACKNOWLEDGMENTS}

We thank Edanz Group (https://en-author-services.edanzgroup. com/ac) for editing the English text of a draft of this manuscript. 


\section{REFERENCES}

Banez-Coronel, M., and Ranum, L. P. W. (2019). Repeat-associated non-AUG (RAN) translation: insights from pathology. Lab. Investig. 99, 929-942. doi: 10.1038/s41374-019-0241-x

Basri, R., Yabe, I., Soma, H., and Sasaki, H. (2007). Spectrum and prevalence of autosomal dominant spinocerebellar ataxia in Hokkaido, the northern island of Japan: a study of 113 Japanese families. J. Hum. Genet. 52, 848-855. doi: 10.1007/s10038-007-0182-x

Cheng, W., Wang, S., Zhang, Z., Morgens, D. W., Hayes, L. R., Lee, S., et al. (2019). CRISPR-Cas9 Screens Identify the RNA Helicase DDX3X as a Repressor of C9ORF72 (GGGGCC)n repeat-associated non-AUG translation. Neuron 104, 885-898.e8. doi: 10.1016/j.neuron.2019.09.003

Courchaine, E. M., Lu, A., and Neugebauer, K. M. (2016). Droplet organelles? EMBO J. 35, 1603-1612. doi: 10.15252/embj.201593517

Eymery, A., Souchier, C., Vourc'h, C., and Jolly, C. (2010). Heat shock factor 1 binds to and transcribes satellite II and III sequences at several pericentromeric regions in heat-shocked cells. Exp. Cell Res. 316, 1845-1855. doi: 10.1016/j. yexcr.2010.02.002

Gasset-Rosa, F., Lu, S., Yu, H., Chen, C., Melamed, Z., Guo, L., et al. (2019). Cytoplasmic TDP-43 de-mixing independent of stress granules drives inhibition of nuclear import, loss of nuclear TDP-43, and cell death. Neuron 102, 339-357.e7. doi: 10.1016/j.neuron.2019.02.038

Gendron, T. F., Bieniek, K. F., Zhang, Y. J., Jansen-West, K., Ash, P. E. A., Caulfield, T., et al. (2013). Antisense transcripts of the expanded C9ORF72 hexanucleotide repeat form nuclear RNA foci and undergo repeat-associated non-ATG translation in c9FTD/ALS. Acta Neuropathol. 126, 829-844. doi: 10.1007/s00401-013-1192-8

Green, K. M., Sheth, U. J., Flores, B. N., Wright, S. E., Sutter, A. B., Kearse, M. G., et al. (2019). High-throughput screening yields several small-molecule inhibitors of repeat-associated non-AUG translation. J. Biol. Chem. 294, 1862418638. doi: 10.1074/jbc.RA119.009951

Guo, L., and Shorter, J. (2015). It's raining liquids: RNA tunes viscoelasticity and dynamics of membraneless organelles. Mol. Cell 60, 189-192. doi: 10.1016/j. molcel.2015.10.006

Hayashi, M., Adachi, Y., Mori, M., Nakano, T., and Nakashima, K. (2007). Clinical and genetic epidemiological study of 16q22.1-linked autosomal dominant cerebellar ataxia in western Japan. Acta Neurol. Scand. 116, 123-127. doi: 10. 1111/j.1600-0404.2007.00815.x

He, F., Krans, A., Freibaum, B. D., Paul Taylor, J., and Todd, P. K. (2014). TDP43 suppresses CGG repeat-induced neurotoxicity through interactions with HnRNP A2/B1. Hum. Mol. Genet. 23, 5036-5051. doi: 10.1093/hmg/ddu216

Ishiguro, T., Sato, N., Ueyama, M., Fujikake, N., Sellier, C., Kanegami, A., et al. (2017). Regulatory role of RNA chaperone TDP-43 for RNA misfolding and repeat-associated translation in SCA31. Neuron 94, 108-124.e7. doi: 10.1016/j. neuron.2017.02.046

Ishikawa, K., Dürr, A., Klopstock, T., Müller, S., De Toffol, B., Vidailhet, M., et al. (2011). Pentanucleotide repeats at the spinocerebellar ataxia type 31 (SCA31) LOCUS IN Caucasians. Neurology 77, 1853-1855.

Ishikawa, K., and Mizusawa, H. (2010). The chromosome 16q-linked autosomal dominant cerebellar ataxia (16q-ADCA): A newly identified degenerative ataxia in Japan showing peculiar morphological changes of the Purkinje cell: the 50th Anniversary of Japanese Society of Neuropathology. Neuropathology 30, 490-494. doi: 10.1111/j.1440-1789.2010.01142.x

Ishikawa, K., and Nagai, Y. (2019). Molecular mechanisms and future therapeutics for spinocerebellar ataxia type 31 (SCA31). Neurotherapeutics 16, 1106-1114. doi: 10.1007/s13311-019-00804-6

Ishiura, H., Doi, K., Mitsui, J., Yoshimura, J., Matsukawa, M. K., Fujiyama, A., et al. (2018). Expansions of intronic TTTCA and TTTTA repeats in benign adult familial myoclonic epilepsy. Nat. Genet. 50, 581-590. doi: 10.1038/s41588-0180067-2

Jain, A., and Vale, R. D. (2017). RNA phase transitions in repeat expansion disorders. Nature 546, 243-247. doi: 10.1038/nature22386

Jin, P., Duan, R., Qurashi, A., Qin, Y., Tian, D., Rosser, T. C., et al. (2007). Pur $\alpha$ Binds to rCGG Repeats and Modulates Repeat-Mediated Neurodegeneration in a Drosophila Model of Fragile X Tremor/Ataxia Syndrome. Neuron 55, 556-564. doi: 10.1016/j.neuron.2007.07.020

Jolly, C., and Lakhotia, S. C. (2006). Human sat III and Drosophila hsrw transcripts: a common paradigm for regulation of nuclear RNA processing in stressed cells. Nucleic Acids Res. 34, 5508-5514. doi: 10.1093/nar/gkl711
Jones, K., Wei, C., Schoser, B., Meola, G., Timchenko, N., and Timchenko, L. (2015). Reduction of toxic RNAs in myotonic dystrophies type 1 and type 2 by the RNA helicase p68/DDX5. Proc. Natl. Acad. Sci.U.S.A. 112, 8041-8045. doi: $10.1073 /$ pnas. 1422273112

Kato, M., Han, T. W., Xie, S., Shi, K., Du, X., Wu, L. C., et al. (2012). Cellfree formation of RNA granules: low complexity sequence domains form dynamic fibers within hydrogels. Cell 149, 753-767. doi: 10.1016/j.cell.2012. 04.017

Kim, D. H., Langlois, M. A., Lee, K. B., Riggs, A. D., Puymirat, J., and Rossi, J. J. (2005). HnRNP H inhibits nuclear export of mRNA containing expanded CUG repeats and a distal branch point sequence. Nucleic Acids Res. 33, 3866-3874. doi: $10.1093 /$ nar/gki698

Kino, Y., Washizu, C., Kurosawa, M., Oma, Y., Hattori, N., Ishiura, S., et al. (2015). Nuclear localization of MBNL1: splicing-mediated autoregulation and repression of repeat-derived aberrant proteins. Hum. Mol. Genet. 24, 740-756. doi: $10.1093 / \mathrm{hmg} / \mathrm{ddu} 492$

Kroschwald, S., Maharana, S., Mateju, D., Malinovska, L., Nüske, E., Poser, I., et al. (2015). Promiscuous interactions and protein disaggregases determine the material state of stress-inducible RNP granules. Elife 4, 1-32. doi: 10.7554/eLife. 06807

Lee, P. H., Park, H. Y., Jeong, S. Y., Hong, J. H., and Kim, H. J. (2007). 16q-linked autosomal dominant cerebellar ataxia in a Korean family [10]. Eur. J. Neurol. 14, 16-17. doi: 10.1111/j.1468-1331.2007.01818.x

Lee, Y. C., Liu, C. S., Lee, T. Y., Lo, Y. C., Lu, Y. C., and Soong, B. W. (2012). SCA31 is rare in the Chinese population on Taiwan. Neurobiol. Aging 33, 426.e23-426.e24. doi: 10.1016/j.neurobiolaging.2010.10.012

Ling, S. C., Polymenidou, M., and Cleveland, D. W. (2013). Converging mechanisms in als and FTD: disrupted RNA and protein homeostasis. Neuron 79, 416-438. doi: 10.1016/j.neuron.2013.07.033

Linsalata, A. E., He, F., Malik, A. M., Glineburg, M. R., Green, K. M., Natla, S., et al. (2019). DDX $3 \mathrm{X}$ and specific initiation factors modulate FMR 1 repeat-associated non-AUG-initiated translation. EMBO Rep. 20:e47498. doi: 10.15252/embr.201847498

Liu, E. Y., Cali, C. P., and Lee, E. B. (2017). RNA metabolism in neurodegenerative disease. DMM Dis. Model. Mech. 10, 509-518. doi: 10.1242/dmm.028613

Lo Piccolo, L., Mochizuki, H., and Nagai, Y. (2019). The lncRNA hsr $\omega$ regulates arginine dimethylation of human FUS to cause its proteasomal degradation in Drosophila. J. Cell Sci. 132:jcs236836. doi: 10.1242/jcs.236836

Lo Piccolo, L., and Yamaguchi, M. (2017). RNAi of arcRNA hsrw affects subcellular localization of Drosophila FUS to drive neurodiseases. Exp. Neurol. 292, 125-134. doi: 10.1016/j.expneurol.2017.03.011

Maharana, S., Wang, J., Papadopoulos, D. K., Richter, D., Pozniakovsky, A., Poser, I., et al. (2018). RNA buffers the phase separation behavior of prion-like RNA binding proteins. Science 360, 918-921. doi: 10.1126/science.aar7366

McGurk, L., Berson, A., and Bonini, N. M. (2015). Drosophila as an in vivo model for human neurodegenerative disease. Genetics 201, 377-402. doi: 10.1534/ genetics.115.179457

Metz, A., Soret, J., Vourc'h, C., Tazi, J., and Jolly, C. (2004). A key role for stressinduced satellite III transcripts in the relocalization of splicing factors into nuclear stress granules. J. Cell Sci. 117, 4551-4558. doi: 10.1242/jcs.01329

Mohan, A., Goodwin, M., and Swanson, M. S. (2014). RNA-protein interactions in unstable microsatellite diseases. Brain Res. 1584, 3-14. doi: 10.1016/j.brainres. 2014.03.039

Molliex, A., Temirov, J., Lee, J., Coughlin, M., Kanagaraj, A. P., Kim, H. J., et al. (2015). Phase separation by low complexity domains promotes stress granule assembly and drives pathological fibrillization. Cell 163, 123-133. doi: 10.1016/ j.cell.2015.09.015

Mori, K., Lammich, S., Mackenzie, I. R. A., Forné, I., Zilow, S., Kretzschmar, H., et al. (2013). HnRNP A3 binds to GGGGCC repeats and is a constituent of p62-positive/TDP43-negative inclusions in the hippocampus of patients with C9orf72 mutations. Acta Neuropathol. 125, 413-423. doi: 10.1007/s00401-0131088-7

Mori, K., Nihei, Y., Arzberger, T., Zhou, Q., Mackenzie, I. R., Hermann, A., et al. (2016). Reduced hn RNPA 3 increases C9orf72 repeat RNA levels and dipeptiderepeat protein deposition. EMBO Rep. 17, 1314-1325. doi: 10.15252/embr. 201541724

Moseley, M. L., Zu, T., Ikeda, Y., Gao, W., Mosemiller, A. K., Daughters, R. S., et al. (2006). Bidirectional expression of CUG and CAG expansion transcripts and intranuclear polyglutamine inclusions in spinocerebellar ataxia type 8. Nat. Genet. 38, 758-769. doi: 10.1038/ng1827 
Nagaoka, U., Takashima, M., Ishikawa, K., Yoshizawa, K., Yoshizawa, T., Ishikawa, M., et al. (2000). A gene on SCA4 locus causes dominantly inherited pure cerebellar ataxia. Neurology 54, 1971-1975. doi: 10.1212/WNL.54.10.1971

Nakamura, K., Yoshida, K., Matsushima, A., Shimizu, Y., Sato, S., Yahikozawa, H., et al. (2017). Natural history of spinocerebellar ataxia Type 31: a 4-Year prospective study. Cerebellum 16, 518-524. doi: 10.1007/s12311-016-0833-6

Nelson, D. L., Orr, H. T., and Warren, S. T. (2013). The unstable repeats-Three evolving faces of neurological disease. Neuron 77, 825-843. doi: 10.1016/j. neuron.2013.02.022

Nguyen, L., Cleary, J. D., and Ranum, L. P. W. (2019). Repeat-associated NonATG translation: molecular mechanisms and contribution to neurological disease. Annu. Rev. Neurosci. 42, 227-247. doi: 10.1146/annurev-neuro070918-050405

Niimi, Y., Takahashi, M., Sugawara, E., Umeda, S., Obayashi, M., Sato, N., et al. (2013). Abnormal RNA structures (RNA foci) containing a penta-nucleotide repeat (UGGAA)n in the Purkinje cell nucleus is associated with spinocerebellar ataxia type 31 pathogenesis. Neuropathology 33, 600-611. doi: 10.1111/neup. 12032

Nozaki, H., Ikeuchi, T., Kawakami, A., Kimura, A., Koide, R., Tsuchiya, M., et al. (2007). Clinical and genetic characterizations of 16q-linked autosomal dominant spinocerebellar ataxia (AD-SCA) and frequency analysis of AD-SCA in the Japanese population. Mov. Disord. 22, 857-862. doi: 10.1002/mds.21443

Onodera, Y. (2006). Clinical features of linked autosomal dominant cerebellar ataxia in Japanese. October 67, 1300-1302.

Ouyang, Y., He, Z., Li, L., Qin, X., Zhao, Y., and Yuan, L. (2012). Spinocerebellar ataxia type 31 exists in Northeast China. J. Neurol. Sci. 316, 164-167. doi: 10.1016/j.jns.2012.02.005

Ouyang, Y., Sakoe, K., Shimazaki, H., Namekawa, M., Ogawa, T., Ando, Y., et al. (2006). 16q-linked autosomal dominant cerebellar ataxia: a clinical and genetic study. J. Neurol. Sci. 247, 180-186. doi: 10.1016/j.jns.2006. 04.009

Owada, K., Ishikawa, K., Toru, S., Ishida, G., Gomyoda, M., Tao, O., et al. (2005). A clinical, genetic, and neuropathologic study in a family with 16q-linked ADCA type III. Neurology 65, 629-632. doi: 10.1212/01.wnl.0000173065.75680.e2

Paulson, H. (2018). Repeat expansion diseases. Handb. Clin. Neurol. 147, 105-123. doi: 10.1007/3-540-29623-9_8503

Pearson, C. E., Edamura, K. N., and Cleary, J. D. (2005). Repeat instability: mechanisms of dynamic mutations. Nat. Rev. Genet. 6, 729-742. doi: 10.1038/ nrg1689

Pedroso, J. L., Abrahao, A., Ishikawa, K., Raskin, S., De Souza, P. V. S., De Rezende Pinto, W. B. V., et al. (2015). When should we test patients with familial ataxias for SCA31? A misdiagnosed condition outside Japan? J. Neurol. Sci. 355, 206-208. doi: 10.1016/j.jns.2015.05.016

Pettersson, O. J., Aagaard, L., Andrejeva, D., Thomsen, R., Jensen, T. G., and Damgaard, C. K. (2014). DDX6 regulates sequestered nuclear CUG-expanded DMPK-mRNA in dystrophia myotonica type 1. Nucleic Acids Res. 42, 71867200. doi: 10.1093/nar/gku352

Rajkowitsch, L., Chen, D., Stampfl, S., Semrad, K., Waldsich, C., Mayer, O., et al. (2007). RNA chaperones, RNA annealers and RNA helicases. RNA Biol. 4, 118-130. doi: 10.4161/rna.4.3.5445

Reddy, K., Cleary, J. D., and Berglund, J. A. (2020). Drug screen tugs at common thread for repeat disorders. Trends Pharmacol. Sci. 41, 71-73. doi: 10.1016/j. tips.2019.12.004

Rhine, K., Vidaurre, V., and Myong, S. (2020). RNA droplets. Annu. Rev. Biophys. 49, 247-265. doi: 10.1146/annurev-biophys-052118-115508

Sato, N., Amino, T., Kobayashi, K., Asakawa, S., Ishiguro, T., Tsunemi, T., et al. (2009). Spinocerebellar ataxia type 31 Is associated with "Inserted" PentaNucleotide Repeats Containing (TGGAA)n. Am. J. Hum. Genet. 85, 544-557. doi: 10.1016/j.ajhg.2009.09.019

Şentürk, M., and Bellen, H. J. (2018). Genetic strategies to tackle neurological diseases in fruit flies. Curr. Opin. Neurobiol. 50, 24-32. doi: 10.1016/j.conb.2017. 10.017
Shibata, T., Nagano, K., Ueyama, M., Ninomiya, K., Hirose, T., Nagai, Y., et al. (2021). Small molecule targeting r(UGGAA)n disrupts RNA foci and alleviates disease phenotype in Drosophila model. Nat. Commun. 12, 1-13. doi: 10.1038/ s41467-020-20487-4

Shin, Y., and Brangwynne, C. P. (2017). Liquid phase condensation in cell physiology and disease. Science 357:eaf4382. doi: 10.1126/science.aaf4382

Simone, R., Balendra, R., Moens, T. G., Preza, E., Wilson, K. M., Heslegrave, A., et al. (2018). G-quadruplex-binding small molecules ameliorate C9orf72 FTD / ALS pathology in vitro and in vivo. EMBO Mol. Med. 10, 22-31. doi: 10.15252/emmm.201707850

Su, Z., Zhang, Y., Gendron, T. F., Bauer, P. O., Chew, J., Yang, W. Y., et al. (2014). Discovery of a biomarker and lead small molecules to target r(GGGGCC)Associated Defects in c9FTD/ALS. Neuron 83, 1043-1050. doi: 10.1016/j. neuron.2014.07.041

Valgardsdottir, R., Chiodi, I., Giordano, M., Cobianchi, F., Riva, S., and Biamonti, G. (2004). Structural and functional characterization of noncoding repetitive RNAs transcribed in stressed human cells. Mol. Biol. Cell 15, 5318-5328. doi: 10.1091/mbc.E04

Valgardsdottir, R., Chiodi, I., Giordano, M., Rossi, A., Bazzini, S., Ghigna, C., et al. (2008). Transcription of Satellite III non-coding RNAs is a general stress response in human cells. Nucleic Acids Res. 36, 423-434. doi: 10.1093/nar/ gkm1056

Weber, S. C., and Brangwynne, C. P. (2012). Getting RNA and protein in phase. Cell 149, 1188-1191. doi: 10.1016/j.cell.2012.05.022

Wilburn, B., Rudnicki, D. D., Zhao, J., Weitz, T. M., Cheng, Y., Gu, X., et al. (2011). An Antisense CAG repeat transcript at JPH3 locus mediates expanded polyglutamine protein toxicity in Huntington's disease-like 2 mice. Neuron 70, 427-440. doi: 10.1016/j.neuron.2011.03.021

Wojciechowska, M., and Krzyzosiak, W. J. (2011). Cellular toxicity of expanded RNA repeats: Focus on RNA foci. Hum. Mol. Genet. 20, 3811-3821. doi: 10. 1093/hmg/ddr299

Wolozin, B. (2019). The evolution of phase-separated TDP-43 in stress. Neuron 102, 265-267. doi: 10.1016/j.neuron.2019.03.041

Yoshida, K., Asakawa, M., Suzuki-Kouyama, E., Tabata, K., Shintaku, M., Ikeda, S., et al. (2014). Distinctive features of degenerating Purkinje cells in spinocerebellar ataxia type 31. Neuropathology 34, 261-267. doi: 10.1111/neup. 12090

Yoshida, K., Shimizu, Y., Morita, H., Okano, T., Sakai, H., Ohata, T., et al. (2009). Severity and Progression Rate of Cerebellar Ataxia in 16q-linked Autosomal Dominant Cerebellar Ataxia (16q-ADCA) in the Endemic Nagano Area of Japan. Cerebellum 8, 46-51. doi: 10.1007/s12311-008-0062-8

Zhang, N., and Ashizawa, T. (2017). RNA toxicity and foci formation in microsatellite expansion diseases. Curr. Opin. Genet. Dev. 44, 17-29. doi: 10. 1016/j.gde.2017.01.005

Zu, T., Gibbens, B., Doty, N. S., Gomes-Pereira, M., Huguet, A., Stone, M. D., et al. (2011). Non-ATG-initiated translation directed by microsatellite expansions. Proc. Natl. Acad. Sci. U.S.A. 108, 260-265. doi: 10.1073/pnas.1013343108

Zu, T., Liu, Y., Bañez-Coronel, M., Reid, T., Pletnikova, O., Lewis, J., et al. (2013). RAN proteins and RNA foci from antisense transcripts in C9ORF72 ALS and frontotemporal dementia. Proc. Natl. Acad. Sci. U.S.A. 110, E4968-E4977. doi: 10.1073/pnas.1315438110

Conflict of Interest: The authors declare that the research was conducted in the absence of any commercial or financial relationships that could be construed as a potential conflict of interest.

Copyright (c) 2021 Ishiguro, Nagai and Ishikawa. This is an open-access article distributed under the terms of the Creative Commons Attribution License (CC BY). The use, distribution or reproduction in other forums is permitted, provided the original author(s) and the copyright owner(s) are credited and that the original publication in this journal is cited, in accordance with accepted academic practice. No use, distribution or reproduction is permitted which does not comply with these terms. 